\title{
Letter
}

\section{The centripetal force law and the equation of motion for a particle on a curved hypersurface}

\author{
L. D. Hu, D. K. Lian, Q. H. Liu \\ School for Theoretical Physics, School of Physics and Electronics, Hunan University, Changsha 410082, China
}

Received: 9 August 2016 / Accepted: 1 November 2016 / Published online: 28 November 2016

(C) The Author(s) 2016. This article is published with open access at Springerlink.com

\begin{abstract}
It is pointed out that the current form of the extrinsic equation of motion for a particle constrained to remain on a hypersurface is in fact a half-finished version; for it is established without regard to the fact that the particle can never depart from the geodesics on the surface. Once this fact is taken into consideration, the equation takes the same form as that for the centripetal force law, provided that the symbols are re-interpreted so that the law is applicable for higher dimensions. The controversial issue of constructing operator forms of these equations is addressed, and our studies show the quantization of constrained system based on the extrinsic equation of motion is preferable.
\end{abstract}

\section{Introduction}

The motion of a particle on a curved hypersurface is an exactly solvable model to examine various problems such as higher-dimensional gravity [1], the dark energy/matter problem [2], the quantization of constrained motions for a nonrelativistic particle [3-7] and for a relativistic fermion [8,9], and many curvature-induced effects in lower-dimensional systems and nanostructures [10-15], etc. We are familiar with both the geodesic equation from the intrinsically curved surface and the equation of motion from the extrinsically Euclidean space [5,6], but no relationship in between has been seriously explored. From the point of geometry, the geodesic equation can be independent from the extrinsic world, but the motion of the particle from the extrinsic view can never be independent from the geodesics that the particle follows.

In an $N$ dimensional Euclidean space $E^{N}$ spanned by $N$ mutually orthogonal unit vectors $\mathbf{e}_{i}(i=1,2, \ldots, N)$, we can embed it as a hypersurface described by a constraint $f(\mathbf{r})=0$ where $f(\mathbf{r})$ is some smooth function of position $\mathbf{r}=x^{i} \mathbf{e}_{i}$ and the Einstein summation convention of sum over

a e-mail: quanhuiliu@gmail.com repeated indices is hereafter assumed. For a non-relativistic particle, with mass $\mu$, that is constrained to remain on the hypersurface, there are two equations of motion for the particle. One is well known, given by the differential equation for a geodesic line $C$ determined by

$\frac{\mathrm{d}^{2} u^{\mu}(s)}{\mathrm{d} s^{2}}+\Gamma_{\alpha \beta}^{\mu} \frac{\mathrm{d} u^{\alpha}(s)}{\mathrm{d} s} \frac{\mathrm{d} u^{\beta}(s)}{\mathrm{d} s}=0$,

where $\left\{u^{\mu}\right\}(\mu=1,2, \ldots, N-1)$ are the $N-1$ local coordinates, and $s$ stands for the arc-length along $C$, and $\Gamma_{\alpha \beta}^{\mu}$ are Christoffel symbols of the second kind. Another one is quite well known [5,6], given by the differential of the velocity $\mathbf{v} \equiv \mathrm{d} \mathbf{r} / \mathrm{d} t$ with respect to time $t$,

$\frac{\mathrm{d}}{\mathrm{d} t} \mathbf{v}=-\mathbf{n}(\mathbf{v} \cdot \nabla \mathbf{n} \cdot \mathbf{v})$,

where $\mathbf{n} \equiv \nabla f(\mathbf{r}) /|\nabla f(\mathbf{r})|$ is the local unit normal vector on the surface at point $\mathbf{r}=\mathbf{r}(u)$, and $\nabla \equiv \mathbf{e}^{i} \partial / \partial x^{i}$ is the usual gradient operator. Though both Eqs. (1) and (2) have the salient feature of not containing the mass $\mu$, the possible difference between them is more interesting and challenging. Some authors claim the two to be identical but no justification is available [5]. In order to obtain a proper form of Eq. (2) in quantum mechanics, it is usually assumed $[6,16]$ that Eq. (2) has a direct correspondence in quantum mechanics once the velocity is rewritten in terms of momentum $\mathbf{v}=\mathbf{p} / \mu$, with possible ordering distributions of the momentum $\mathbf{p}$ and position-dependent functions $\nabla \mathbf{n}$ and $\mathbf{n}$, in the Heisenberg picture,

$\frac{\mathrm{d}}{\mathrm{d} t} \mathbf{p}=-\mathbf{n}\left(\frac{\mathbf{p} \cdot \nabla \mathbf{n} \cdot \mathbf{p}}{\mu}\right)$.

However, on the one hand, Eq. (1) is purely from intrinsic geometry, from which we know that the Dirac quantization of the constrained systems cannot be fulfilled throughout [17]. On the other hand, Eq. (3) contains mutually dependent components of the momentum $\mathbf{p}$ because the motion lies on the tangential plane to the surface so that $\mathbf{n} \cdot \mathbf{p}=0$. Thus in 
quantum mechanics, we have inequivalent forms of (3) [16]. In other words, Eq. (1) under-describes the motion of the particle and must be enlarged, while Eq. (3) over-describes it; it must be used with some constraints. Therefore, a proper form of the equation of motion for the particle in classical mechanics is worthy to be investigated. In quantum mechanics the meaning of Eq. (3) is under dispute. For instance, Hamma et al. [4] and Ikegami et al. [5] showed that from the constraint equation $f(\mathbf{r})=0$ we could not build up a satisfactory theory and we must start from another constraint equation $\mathrm{d} f(\mathbf{r}) / \mathrm{d} t=0$, but Weinberg [6] thought that Eq. (3) should be as true as it is in classical mechanics. ${ }^{1}$

In Sect. 2, the generalized form of the centripetal force law (CFL) which incorporates both Eqs. (1) and (3) is given. In Sect. 3, the quantization problem of the constrained motion on the surface is addressed. Though there is no explicit use of the Dirac formalism for a constrained system, our explorations are in fact within it because we further develop the results (1)-(3) that are based on the Dirac formalism. In Sect. 4 , a brief conclusion and discussion are presented.

\section{The generalized CFL unifies both intrinsic and extrinsic equation of motion}

For our purpose, let us first recall the celebrated CFL $a=$ $v^{2} / r$ for the particle moving on a planar curve, especially on the $2 D$ circle of radius $r$, and it can readily be rewritten in terms of the curvature $\kappa(\equiv 1 / r)$ and the Hamiltonian $H \equiv$ $p^{2} / 2 \mu=\mu v^{2} / 2$ for the free motion without any external force imposed,

$\frac{\mathrm{d}}{\mathrm{d} t} \mathbf{p}=-2 H \kappa \mathbf{n}$.

In fact, Eq. (4) holds true in general provided that $\kappa$ symbolizes the first curvature of the geodesic $C$ on the hypersurface and the Hamiltonian $H$ applies to the free particle on the surface.

From the differential geometry for the hypersurface, at the point $\left\{u^{\mu}\right\}$ on the surface $f(\mathbf{r})=0$, we can define the vectors of the tangential space $\mathbf{r}_{\alpha}\left(\equiv \mathrm{d} \mathbf{r} / \mathrm{d} u^{\alpha}\right)$ and the unit normal vector $\mathbf{n}$, and these vectors $\left\{\mathbf{r}_{\alpha}, \mathbf{n}\right\}$ form a complete set of the coordinates in the vicinity of the surface in the $E^{N}$, other than the orthogonal coordinates formed by the

\footnotetext{
${ }^{1}$ Compare the formula (9.5.30) in [6], which is in fact identical to Eq. (3) when $V=0$. In the first edition of the book, this formula is mistaken. It is worthy of pointing out that many formulas in this section of the first edition were seriously mistaken, including, for instance, (9.5.5), (9.5.7), (9.5.28) and (9.5.30). One of the present authors (Q. H. Liu) pointed out some mistakes to the author Professor Weinberg who then performed an overall examination of the whole section with great care and acknowledged him in the Addendum for the Second Edition though misspelling his name as Lu Quanhui (the full name in Chinese order should be Liu Quanhui).
}

fixed Cartesian one $\left\{\mathbf{e}_{i}\right\}$. The first and second fundamental quantities are $g_{\alpha \beta} \equiv \mathbf{r}_{\alpha} \cdot \mathbf{r}_{\beta}$ and $b_{\alpha \beta} \equiv \mathbf{r}_{\alpha \beta} \cdot \mathbf{n}=-\mathbf{r}_{\alpha} \cdot \mathbf{n}_{\beta}$, respectively. The equations of motion for $\mathbf{r}_{\alpha}$ and $\mathbf{n}$ are in the $E^{N}$ [18], respectively,

$$
\begin{aligned}
& \frac{\partial \mathbf{r}_{\alpha}}{\partial u^{\beta}}=\Gamma_{\alpha \beta}^{\mu} \mathbf{r}_{\mu}+b_{\alpha \beta} \mathbf{n}, \\
& \frac{\partial \mathbf{n}}{\partial u^{\alpha}}=-b_{\alpha}^{\beta} \mathbf{r}_{\beta} .
\end{aligned}
$$

Furthermore, from the differential geometry for curves $\mathbf{r}(s)$ lying on the surface, we can define, respectively, the unit tangential $\alpha$ and its derivative with respect to $s$ as follows:

$$
\begin{aligned}
& \alpha \equiv \frac{\mathrm{d} \mathbf{r}(s)}{\mathrm{d} s}=\frac{\partial \mathbf{r}}{\partial u^{\alpha}} \frac{\mathrm{d} u^{\alpha}}{\mathrm{d} s} \equiv \mathbf{r}_{\alpha} \frac{\mathrm{d} u^{\alpha}}{\mathrm{d} s}, \\
& \frac{\mathrm{d}^{2} \mathbf{r}}{\mathrm{d} s^{2}}=\frac{\mathrm{d} \alpha}{\mathrm{d} s}=\frac{\mathrm{d}}{\mathrm{d} s}\left(\mathbf{r}_{\alpha} \frac{\mathrm{d} u^{\alpha}}{\mathrm{d} s}\right) .
\end{aligned}
$$

First, we limit the curve $\mathbf{r}(s)$ to be the geodesic line $C$. On the one hand, the first curvature $\kappa$ is defined by [18]

$$
\frac{\mathrm{d}^{2} \mathbf{r}}{\mathrm{d} s^{2}}=\kappa \mathbf{m},
$$

where the vector $\mathbf{m}$ is a unit normal vector of the curve, which by the convention of the geometry is identical to $-\mathbf{n}[18,19]$. On the other hand, the right-handed side of Eq. (8) becomes, from (5) and (1),

$$
\begin{aligned}
\frac{\mathrm{d}}{\mathrm{d} s} & \left(\mathbf{r}_{\alpha} \frac{\mathrm{d} u^{\alpha}}{\mathrm{d} s}\right)=\frac{\partial \mathbf{r}_{\alpha}}{\partial u^{\beta}} \frac{\mathrm{d} u^{\alpha}}{\mathrm{d} s} \frac{\mathrm{d} u^{\beta}}{\mathrm{d} s}+\mathbf{r}_{\alpha} \frac{\mathrm{d}^{2} u^{\alpha}(s)}{\mathrm{d} s^{2}} \\
= & \left(\Gamma_{\alpha \beta}^{\mu} \mathbf{r}_{\mu}+b_{\alpha \beta} \mathbf{n}\right) \frac{\mathrm{d} u^{\alpha}}{\mathrm{d} s} \frac{\mathrm{d} u^{\beta}}{\mathrm{d} s} \\
& -\Gamma_{\alpha \beta}^{\mu} \frac{\mathrm{d} u^{\alpha}(s)}{\mathrm{d} s} \frac{\mathrm{d} u^{\beta}(s)}{\mathrm{d} s} \mathbf{r}_{\mu} \\
= & b_{\alpha \beta} \frac{\mathrm{d} u^{\alpha}}{\mathrm{d} s} \frac{\mathrm{d} u^{\beta}}{\mathrm{d} s} \mathbf{n} .
\end{aligned}
$$

Substituting (9) and (12) into both sides of (8), we get, noting $\mathbf{m}=-\mathbf{n}$, the relation between curvature $\kappa$ and the second fundamental quantities $b_{\alpha \beta}$,

$\kappa=-b_{\alpha \beta} \frac{\mathrm{d} u^{\alpha}}{\mathrm{d} s} \frac{\mathrm{d} u^{\beta}}{\mathrm{d} s}$.

We see that the curvature $\kappa$ of the curve is related to the second fundamental quantities, the extrinsic geometric ones, of the surface. In geometry, $-\kappa \mathbf{n}$ is a geometric invariant under the parameter transformation $\left\{u^{\alpha}\right\} \rightarrow\left\{u^{\prime \alpha}\right\}$.

Secondly, taking the derivative of the orthogonal relation $\mathbf{n} \cdot \mathbf{r}_{\gamma}=0$ with respect to any local coordinate $u^{\alpha}$, noting $\mathbf{n}=\mathbf{n}(\mathbf{r}(u))$, we find

$$
\begin{aligned}
0=\frac{\partial}{\partial u^{\alpha}}\left(\mathbf{n} \cdot \mathbf{r}_{\gamma}\right) & =\mathbf{r}_{\alpha} \cdot \nabla \mathbf{n} \cdot \mathbf{r}_{\gamma}+\mathbf{n} \cdot \frac{\partial}{\partial u^{\alpha}} \mathbf{r}_{\gamma} \\
& =\mathbf{r}_{\alpha} \cdot \nabla \mathbf{n} \cdot \mathbf{r}_{\gamma}+b_{\alpha \gamma}
\end{aligned}
$$


i.e.

$b_{\alpha \gamma}=-\mathbf{r}_{\alpha} \cdot \nabla \mathbf{n} \cdot \mathbf{r}_{\gamma}$.

Substituting it into Eq. (13), we have another form of the curvature $\kappa$,

$$
\begin{aligned}
\kappa & =-b_{\alpha \beta} \frac{\mathrm{d} u^{\alpha}}{\mathrm{d} s} \frac{\mathrm{d} u^{\beta}}{\mathrm{d} s}=\mathbf{r}_{\beta} \cdot \nabla \mathbf{n} \cdot \mathbf{r}_{\alpha} \frac{\mathrm{d} u^{\alpha}}{\mathrm{d} s} \frac{\mathrm{d} u^{\beta}}{\mathrm{d} s} \\
& =\frac{\mathrm{d} \mathbf{r}}{\mathrm{d} s} \cdot \nabla \mathbf{n} \cdot \frac{\mathrm{d} \mathbf{r}}{\mathrm{d} s} .
\end{aligned}
$$

Third, since $\alpha=d \mathbf{r} / d s$ is the unit tangential vector along the curve $C$ and so is the ratio $\mathbf{p} / p=\mathbf{v} / v$, the expression $\mathbf{p} \cdot \nabla \mathbf{n} \cdot \mathbf{p} / \mu$ can be written within the framework of the Hamiltonian mechanics and differential geometry,

$$
\begin{aligned}
\frac{\mathbf{p} \cdot \nabla \mathbf{n} \cdot \mathbf{p}}{\mu} & =\left(\frac{\mathbf{p}}{p} \cdot \nabla \mathbf{n} \cdot \frac{\mathbf{p}}{p}\right) \frac{p^{2}}{\mu}=\left(\frac{\mathrm{d} \mathbf{r}}{\mathrm{d} s} \cdot \nabla \mathbf{n} \cdot \frac{\mathrm{d} \mathbf{r}}{\mathrm{d} s}\right) \frac{p^{2}}{\mu} \\
& =2 \kappa H .
\end{aligned}
$$

Substituting it into (3), we obtain Eq. (4). Thus, the CFL holds true universally.

For the particle that is constrained on an arbitrary $N-$ 1 dimensional space curve $f(\mathbf{r}(u(s)))=0$, we can prove the CFL (4) as well. Under the coordinate transformation $\left\{u^{\alpha}\right\} \rightarrow\left\{u^{\prime \alpha}\right\}\left(\neq\left\{u^{\alpha}\right\}\right)$, the two equations (1) and (4) are completely different for the former transform accordingly whereas the latter keeps invariant. In other words, Eq. (4) is geometric invariant whereas Eq. (1) is not, though both are covariant.

\section{Remarks on the quantization problem of the constrained motion}

In quantum mechanics, no momentum $\mathbf{p}=\left\{p_{x}, p_{y}, p_{z}\right\}$ could be taken to substitute into the term $\left(p_{x}^{2}+p_{y}^{2}+p_{z}^{2}\right) / 2 \mu$ so as to get the satisfactory quantum-mechanical operator $H=-\hbar^{2} \nabla_{L B}^{2} / 2 \mu+V_{q}$ where $V_{q} \equiv-\frac{\hbar^{2}}{2 \mu}\left(\frac{1}{4}\left(2 \operatorname{Tr}(\mathbf{k})^{2}-\right.\right.$ $\left.\left.(\operatorname{Tr} \mathbf{k})^{2}\right)\right)$ is the geometric potential obtained by the confining potential technique [20], with $\mathbf{k}$ being the extrinsic curvature tensor $[3,5]$. This curvature-induced potential has been confirmed by experiments $[21,22]$ and may play some role in understanding of our present universe [2]. An illuminative exploration is to compare both sides of Eq. (3) in quantum mechanics,

$[\mathbf{p}, H]=\frac{i \hbar}{\mu} O[\mathbf{p} \cdot \nabla \mathbf{n} \cdot \mathbf{p}]$,

where $H$ is the quantum-mechanical Hamiltonian containing the geometric potential, and $O[f]$ stands for the hermitian operator of the quantity $f$. Equation (18) allows for both the geometric momentum $\mathbf{p}$ [20] and the geometric potential $V_{q}$, and the geometric momentum is given by $\mathbf{p}=i \frac{\mu}{\hbar}[H, \mathbf{r}]=-i \frac{\hbar}{2}\left[\nabla_{L B}^{2}, \mathbf{r}\right]=-i \hbar\left(\nabla_{S}+\frac{M \mathbf{n}}{2}\right)$,

where the vector operator $\nabla_{S} \equiv \mathbf{r}^{\mu} \partial_{\mu}$ is the gradient operator defined on the surface, and $-\nabla_{S} \cdot \mathbf{n}=M$ is the mean curvature [2]. In contrast, the operator $O[\mathbf{p} \cdot \nabla \mathbf{n} \cdot \mathbf{p}]$ in (18) gives us many choices as regards the dependence on the components of position and momentum [16], because we are free to choose not only independent coordinates but also momenta; for we have two constrained conditions $f(\mathbf{r})=0$ and $\mathbf{n} \cdot \mathbf{p}=0$. It may not be a shortcoming, though. Instead, the over-description has the remarkable advantage to include the results predicted by the the confining potential technique $[2,3,5,16,17,20,23-25]$.

Thus, from the point of view of the operator algebra, a complete formulation of the quantization of the constrained motion is still an open problem $[4,26]$. Our approach supports Weinberg, whose point is that Eq. (3) holds true in quantum mechanics [6], and disfavors Homma et al. [4] and Ikegami et al. [5], whose point is that the satisfactory theory from the constraint equation $f(\mathbf{r})=0$ is impossible to obtain with the Dirac formalism for quantizing a constrained system. Besides, the statement [5] that Eq. (3) is identical to Eq. (1) is nevertheless wrong.

However, the quantum mechanical version of CFL (4),

$[\mathbf{p}, H]=i \hbar(\kappa \mathbf{n} H+H \kappa \mathbf{n})$,

is not applicable unless for very special surfaces such as the spherical one [2]. This is because this equation contains the first curvature $\kappa$ of the geodesic curve, which represents a classical orbit, and its operator version is hard to verify except when the curvature is a constant. For an $N-1$ dimensional sphere whose $\kappa=1 / r$, with $r$ being radius, Eq. (20) predicts an additional energy of $V_{q}=(N-1)(N-3) \hbar^{2} / 8 m r^{2}$, which is exactly the geometric potential for the sphere [2].

\section{Conclusions and discussions}

The study of the CFL can be traced back to Newton in the 17 th century, which was a crucial step toward his law of universal gravitation. In classical mechanics, it is the gravity that provides the centripetal force responsible for astronomical orbits. However, in modern physics, there is no gravity but the curved space-time, and the astronomical orbits are nothing but geodesics in it. However, the general relationship between the CFL and the geodesics has been unknown. We show that the relationship is surprisingly simple because the usual form of the CFL is universally applicable provided the meaning of the symbols in it is reinterpreted. Therefore, even as there is no gravity but geodesics on a 
curved space, there is a force law that bears striking resemblance to the CFL. However, in quantum mechanics, even though the operator equation corresponding the CFL (4) has a limited meaning, the equation corresponding to (3) opens a wider door to establishing a satisfactory quantum theory within the Dirac formalism for quantizing a constrained system.

It is well known that within the Dirac formalism there is at least no straightforward way starting from the constraint equation $f(\mathbf{r})=0$ to reach the geometric potential, and some even believe that such a way does not exist. We are convinced that the Dirac formalism is not fully understood yet, and there is a way within the Dirac formalism to begin with $f(\mathbf{r})=0$ to get to the geometric potential, which is under intensive investigations.

Acknowledgements This work is financially supported by National Natural Science Foundation of China under Grant No. 11675051.

Open Access This article is distributed under the terms of the Creative Commons Attribution 4.0 International License (http://creativecomm ons.org/licenses/by/4.0/), which permits unrestricted use, distribution, and reproduction in any medium, provided you give appropriate credit to the original author(s) and the source, provide a link to the Creative Commons license, and indicate if changes were made. Funded by SCOAP ${ }^{3}$.

\section{References}

1. P. Mittelstaedt, J.B. Barbour, Z. Phys. A 203, 82-90 (1967)

2. Q.H. Liu, J. Math. Phys. 54, 122113 (2013)

3. R.C.T. da Costa, Phys. Rev. A 23, 1982-1987 (1981)
4. T. Homma, T. Inamoto, T. Miyazaki, Phys. Rev. D 42, 2049 (1990)

5. M. Ikegami, Y. Nagaoka, S. Takagi, T. Tanzawa, Prog. Theor. Phys. 88, 229 (1992)

6. S. Weinberg, Lectures on Quantum Mechanics, 2nd edn. (Cambridge University Press, Cambridge, 2015), pp. 335-340

7. C. Ortix, Phys. Rev. B 91, 245412 (2015)

8. M. Burgess, B. Jensen, Phys. Rev. A 48, 1861 (1993)

9. F.T. Brandt, J.A. Sánchez-Monroy, Phys. Lett. A 380, 3036-3043 (2016)

10. C. Filgueiras, F. Moraes, Ann. Phys. 323, 3150-3157 (2008)

11. K.V.R.A. Silva, C.F. de Freitas, C. Filgueiras, Eur. Phys. J. B 86, 147 (2013)

12. A. Poux, L.R.S. Araújo, C. Filgueiras, F. Moraes, Eur. Phys. J. Plus 129, 100 (2014)

13. C. Filgueiras, E.O. Silva, Phys. Lett. A 379, 2110-2115 (2015)

14. D. Schmeltzer, J. Phys. Condens. Matter 23, 155601 (2011)

15. V. Atanasov, A. Saxena, J. Phys. Condens. Matter 23, 175301 (2011)

16. Q.H. Liu, J. Zhang, D.K. Lian, L.D. Hu, Z. Li, Generalized centripetal force law and quantization of motion constrained on 2D surfaces. To appear in Physica E Low Dimens Syst Nanostruct (2016). arXiv:1604.05065 [quant-ph]. doi:10.1016/j.physe.2016. 11.029

17. D.M. Xun, Q.H. Liu, Ann. Phys. 341, 132-141 (2014)

18. B. Csikós, Differential Geometry (Typotex Publishing House, Budapest, 2014). Available online: http://etananyag.ttk.elte.hu/ FiLeS/downloads/_01_Csikos_Differential_geometry.pdf

19. M. Spivak, A Comprehensive Introduction to Differential Geometry, vol. 4, 3rd edn. (Publish or Perish, Houston, 1999)

20. Q.H. Liu, L.H. Tang, D.M. Xun, Phys. Rev. A 84, 042101 (2011)

21. A. Szameit et al., Phys. Rev. Lett. 104, 150403 (2010)

22. J. Onoe, T. Ito, H. Shima, H. Yoshioka, S. Kimura, Europhys. Lett. 98, 27001 (2012)

23. Q.H. Liu, J. Phys. Soc. Jpn. 82, 104002 (2013)

24. D.M. Xun, Q.H. Liu, X.M. Zhu, Ann. Phys. 338, 123 (2013)

25. Z.S. Zhang, S.F. Xiao, D.M. Xun, Q.H. Liu, Commun. Theor. Phys. 63, 19 (2015)

26. L. Kaplan, N.T. Maitra, E.J. Heller, Phys. Rev. A 56, 2592 (1997) 